\title{
ABC Transporter Binding
}

National Cancer Institute

\section{Source}

National Cancer Institute. ABC Transporter Binding. NCI Thesaurus. Code C40457.

ABC T ransporter Binding involves temporary non-covalent interaction of a molecule through intermolecular physical forces of attraction with the ABC Transporter complex to move the substrate across the plasma cell membrane against a concentration gradient. 